\title{
Improving workforce scheduling using artificial neural networks model
}

\author{
Simeunović, N. ${ }^{a}$, Kamenko, I. ${ }^{a}$, Bugarski, V. ${ }^{a}$, Jovanović, M. ${ }^{a}$, Lalić, B. ${ }^{a}{ }^{\text {* }}$ \\ ${ }^{a}$ University of Novi Sad, Faculty of Technical Sciences, Novi Sad, Serbia
}

\begin{abstract}
A B S T R A C T
This paper demonstrates a decision support tool for workforce planning and scheduling. The research conducted in this study is oriented on batch type production typical for smaller production systems, workshops and service systems. The derived model in the research is based on historical data from Public utility service billing company. Model uses Artificial Neural Networks (ANN) fitting techniques. A set of eight input indicators is designed and two variants were tested in the model with two different outputs. Several comprehensive parameter setting experiments were performed to improve prediction performances. Real case studies using historic data from public weather database and communal consolidated billing service show that it is difficult to predict the required number of servers-workers in front office. In a similar way, this model is adequate for complex production systems with unpredictable and volatile demand. Therefore, manufacturing systems which create short cycle products, typical for food processing industry, or production for inventory, may benefit of the research presented in this paper. ANN simulation model with its unique set of features and chosen set of training parameters illustrate that presented model may serve as a valuable decision support system in workforce scheduling for service and production systems.
\end{abstract}

\section{ARTICLE INFO}

Keywords:

Workforce scheduling

Production planning

ANN prediction

Operations management

*Corresponding author:

blalic@uns.ac.rs

(Lalić, B.)

Article history:

Received 6 June 2017

Revised 15 September 2017

Accepted 8 November 2017

\section{References}

[1] Aickelin, U., Dowsland, K.A. (2004). An indirect genetic algorithm for a nurse-scheduling problem, Computers \& Operations Research, Vol. 31, No. 5, 761-778, doi: 10.1016/S0305-0548(03)00034-0.

[2] Valls, V., Pérez, Á., Quintanilla, S. (2009). Skilled workforce scheduling in service centres, European Journal of Operational Research, Vol. 193, No. 3, 791-804, doi: 10.1016/j.ejor.2007.11.008.

[3] Bayraktar, E., Jothishankar, M.C., Tatoglu E., Wu, T. (2007). Evolution of operations management: Past, present and future, Management Research News, Vol. 30, No. 11, 843-871, doi: 10.1108/01409170710832278.

[4] Chopra, S., Lovejoy, W., Yano, C. (2004). Five decades of operations management and the prospects ahead, Management Science, Vol. 50, No. 1, 8-14, doi: $10.1287 /$ mnsc.1030.0189.

[5] Sandmann, W. (2013). Quantitative fairness for assessing perceived service quality in queues, Operational Research, Vol. 13, No. 2, 153-186, doi: 10.1007/s12351-011-0111-9.

[6] Calabrese, A., Capece, G., Costa, R., Di Pillo, F., Paglia, D. (2013). The impact of workforce management systems on productivity and quality, Knowledge and Process Management, Vol. 20, No. 3, 177-184, doi: 10.1002/kpm.1417.

[7] Calabrese, A. (2012). Service productivity and service quality: A necessary trade-off?, International Journal of Production Economics, Vol. 135, No. 2, 800-812, doi: 10.1016/j.ijpe.2011.10.014.

[8] Van den Bergh, J., Beliën, J., De Bruecker, P., Demeulemeester, E., De Boeck, L. (2013). Personnel scheduling: A literature review, European Journal of Operational Research, Vol. 226, No. 3, 367-385, doi: 10.1016/j.ejor. 2012. $\underline{11.029}$.

[9] Taylor, D.L., Brunt, D. (2001). Manufacturing operations and supply chain management: The LEAN approach, Thomson Learning, London, UK. 
[10] Mirrazavi, S.K., Beringer, H. (2007). A web-based workforce management system for Sainsburys supermarkets Ltd., Annals of Operations Research, Vol. 155, No. 1, 437-457, doi: 10.1007/s10479-007-0204-2.

[11] Akjiratikarl, C., Yenradee, P., Drake, P.R. (2007). PSO-based algorithm for home care worker scheduling in the UK, Computers \& Industrial Engineering, Vol. 53, No. 4, 559-583, doi: 10.1016/i.cie.2007.06.002.

[12] Mladenović, I., Sokolov-Mladenović, S., Milovančević, M., Marković, D., Simeunović, N. (2016). Management and estimation of thermal comfort, carbon dioxide emission and economic growth by support vector machine, $R e$ newable and Sustainable Energy Reviews, Vol. 64, 466-476, doi: 10.1016/i.rser.2016.06.034.

[13] Rebai, M., Kacem, I., Adjallah, K.H. (2000). Scheduling jobs and maintenance activities on parallel machines, Operational Research, Vol. 13, No. 3, 363-383, doi: 10.1007/s12351-012-0130-1.

[14] Nissen, V., Günther, M. (2009). Staff scheduling with particle swarm optimisation and evolution strategies, In: EvoCOP, Evolutionary Computation in Combinatorial Optimization, Tübingen, Germany, 228-239, doi: 10.1007/ 978-3-642-01009-5 20.

[15] Pinedo, M., Chao, X. (1998). Operations scheduling with applications in manufacturing and services, Irwin /McGraw-Hill, Boston, USA.

[16] Bakhrankova, K. (2008). Production planning in continuous process industries: Theoretical and optimization issues, In: Operations Research Proceedings 2008, University of Augsburg, Augsburg, Germany, 67-72.

[17] ATOSS Software AG, Standort Deutschland 2006: Zukunftssicherung durch intelligentes personal management, from: https://www.atoss.com/en-gb/Workforce-Management, accessed October $13^{\text {th }}, 2016$.

[18] Schroeder, R.G. (1989). Operations management: Decision making in the operations function, McGraw-Hill, New York, USA.

[19] Solomon, M.R., Surprenant, C., Czepiel, J.A., Gutman, E.G. (1985). A role theory perspective on dyadic interactions: The service encounter, Journal of Marketing, Vol. 49, No. 1, 99-111, doi: 10.2307/1251180.

[20] ISO - International Organization for Standardization, ISO 9000:2015, from: https://www.iso.org/standard/ 45481.html, accessed October $13^{\text {th }}, 2016$.

[21] Slack, N., Brandon-Jones, A., Johnston, R. (2014). Operations Management, 7th Edition, Pearson, London, UK.

[22] Vargo, S.L., Lusch, R.F. (2004) The four service marketing myths: Remnants of a goods-based manufacturing model, Journal of Service Research, Vol. 6, No. 4, 324-335, doi: 10.1177/1094670503262946.

[23] Tom, G., Lucey, S. (1995). Waiting time delays and customer satisfaction in supermarkets, Journal of Services Marketing, Vol. 9, No. 5, 20-29, doi: 10.1108/08876049510100281.

[24] Zemke, R. (2002). Managing the employee connection, Managing Service Quality: An International Journal, Vol. 12, No. 2, 73-76, doi: 10.1108/09604520210421374.

[25] Aaker, D.A., Biel, A.L. (1993). Converting image into equity, In: Brand Equity \& Advertising: Advertising's Role in Building Strong Brands, Hillsdale, New York USA, 67-82.

[26] Kerin, R.A., Ambuj, J., Howard, D.J. (1992). Store shopping experience and consumer price-quality-value perceptions, Journal of Retailing, Vol. 68, No. 4, 376-397.

[27] Grace, D., O'Cass, A. (2004). Examining service experiences and post-consumption evaluations, Journal of Services Marketing, Vol. 18, No. 6, 450-461, doi: 10.1108/08876040410557230.

[28] Ren, X.Y., Kong, Z.F., Liang W.C., Li, H.C., Zhou, X.Y. (2017). Vehicle scheduling based on plant growth simulation algorithm and distribution staff behaviour, Advances in Production Engineering \& Management, Vol. 12, No. 2, 173-184, doi: 10.14743/apem2017.2.249.

[29] Bielen, F., Demoulin, N. (2007). Waiting time influence on the satisfaction-loyalty relationship in services, Managing Service Quality: An International Journal, Vol. 17, No. 2, 174-193, doi: 10.1108/09604520710735182.

[30] Yang, Y., Hou, Y., Wang, Y. (2013). On the development of public-private partnerships in transitional economies: An explanatory framework, Public Administration Review, Vol. 73, No. 2, 301-310, doi: 10.1111/j.1540-6210. 2012.02672.x.

[31] Thompson, G.M., Goodale, J.C. (2006). Variable employee productivity in workforce scheduling, European Journal of Operational Research, Vol. 170, No. 2, 376-390, doi: 10.1016/i.ejor.2004.03.048.

[32] Hill, T., Marquez, L., O'Connor, M., Remus, W. (1994). Artificial neural network models for forecasting and decision making, International Journal of Forecasting, Vol. 10, No. 1, 5-15, doi: 10.1016/0169-2070(94)90045-0.

[33] Sundari. M.S., Palaniammal, S. (2015). Simulation of M/M/1 queuing system using ANN, Malaya Journal of Matematik, Vol. 3, No. 1, 279-294.

[34] Altiparmak, F., Dengiz, B., Bulgak, A.A. (2007). Buffer allocation and performance modeling in asynchronous assembly system operations: An artificial neural network metamodeling approach, Applied Soft Computing, Vol. 7, No. 3, 946-956, doi: 10.1016/i.asoc.2006.06.002.

[35] Gul, M., Guneri, A.F. (2015). Forecasting patient length of stay in an emergency department by artificial neural networks, Journal of Aeronautics and Space Technologies, Vol. 8, No. 2, 43-48, doi: 10.7603/s40690-015-0015-7.

[36] Candan, G., Taşkin, M.F., Yazgan, H.R. (2014). Demand forecasting in pharmaceutical industry using artificial intelligence: Neuro-fuzzy approach, Journal of Military and Information Science, Vol. 2, No. 2, 41-49.

[37] Milovic, B., Milovic M. (2012). Prediction and decision making in health care using data mining, International Journal of Public Health Science, Vol. 1, No. 2, 69-78, doi: 10.11591/ijphs.v1i2.1380.

[38] Asensio-Cuesta, S., Diego-Mas, J.A., Canós-Darós, L., Andrés-Romano, C. (2012). A genetic algorithm for the design of job rotation schedules considering ergonomic and competence criteria, The International Journal of Advanced Manufacturing Technology, Vol. 60, No. 9-12, 1161-1174, doi: 10.1007/s00170-011-3672-0.

[39] Carmen, R., Defraeye, M., Van Nieuwenhuyse, I. (2015). A decision support system for capacity planning in emergency departments, International Journal of Simulation Modelling, Vol. 14, No. 2, 299-312, doi: 10.2507/ IJSIMM14(2)10.308.

[40] Baesler, F., Gatica, J., Correa, R. (2015). Simulation optimisation for operating room scheduling, International Journal of Simulation Modelling, Vol. 14, No. 2, 215-226, doi: 10.2507/IJSIMM14(2)3.287. 
[41] García-Alcaraz, J.L., Maldonado-Macías, A.A., Alor-Hernández, G., Sánchez-Ramírez, C. (2017). The impact of information and communication technologies (ICT) on agility, operating, and economical performance of supply chain, Advances in Production Engineering \& Management, Vol. 12, No. 1, 29-40, doi: 10.14743/apem2017.1.237.

[42] Oliver, J.E. (2005). Encyclopedia of world climatology, Springer, Dordrecht, The Netherlands. 
APEM
Advances in Production Engineering \& Management Letnik 12 | Številka 4 | December 2017 | Strani 337-352 https://doi.org/10.14743/apem2017.4.262
ISSN 1854-6250

Spletna stran: apem-journal.org Izvirni znanstveni članek

\title{
Izboljšanje razporejanja delovne sile z modelom umetnih nevronskih mrež
}

\author{
Simeunović, N. ${ }^{a}$, Kamenko, I. ${ }^{a}$, Bugarski, V. ${ }^{a}$, Jovanović, M. ${ }^{a}$, Lalić, B. ${ }^{a,}$ \\ ${ }^{a}$ University of Novi Sad, Faculty of Technical Sciences, Novi Sad, Serbia
}

\section{POVZETEK}

Prispevek predlaga orodje za podporo pri načrtovanju in razporejanju delovne sile. Raziskava, opravljena v tej študiji, je usmerjena na serijsko proizvodnjo, značilno za manjše proizvodne obrate, delavnice in storitvena podjetja. Izpeljani model temelji na podatkih družbe za obračunavanje komunalnih storitev in tehniki umetnih nevronskih mrež (ANN). Zasnovan model ima osem vhodnih indikatorjev ter dve različici z dvema različnima izhodoma. Za izboljšanje napovedovanja smo izvedli več obsežnih poskusov nastavljanja parametrov. Realne študije primerov, ki uporabljajo podatke iz javne vremenske baze in podjetja za obračunavanje komunalnih storitev kažejo, da je težko napovedati število zaposlenih v operativni pisarni. Predlagan pristop je v osnovi primeren tudi za kompleksne proizvodne sisteme z nepredvidljivim in nestanovitnim povpraševanjem. Zato lahko rezultati predstavljeni v tej raziskavi koristijo proizvodnim podjetjem, ki izdelujejo izdelke v kratkem ciklu, značilnem za živilsko predelovalno industrijo ali proizvodnjo na zaloge. Simulacijski model ANN s posebnim naborom značilnosti in parametri za učenje je pokazal, da lahko predstavljeni model služi kot dragoceno orodje pri načrtovanju razporeda delovne sile $\mathrm{v}$ storitvenem in proizvodnem sektorju.

\section{PODATKI O ČLANKU}

Ključne besede:

Razporejanje delovne sile

Načrtovanje proizvodnje

Napovedovanje z ANN

Vodenje operacij

*Kontaktna oseba:

blalic@uns.ac.rs

(Lalić, B.)

Zgodovina članka:

Prejet 6. junija 2017

Popravljen 15. septembra 2017

Sprejet 8. novembra 2017 